Arq. Bras. Med. Vet. Zootec., v.65, n.2, p.364-368, 2013

\title{
Intoxicação crônica por cobre em ovinos: conduta para o diagnóstico conclusivo
}

\author{
[Chronic copper toxicities in sheep: conduct for diagnostic confirmation] \\ M.P. Miguel ${ }^{1}$, M.A. Souza ${ }^{2}$, P.H.J. Cunha ${ }^{3}$, G.L. Costa ${ }^{3}$, L.J. Abud ${ }^{4}$ \\ ${ }^{1}$ Universidade Federal de Goiás - Jataí, GO \\ ${ }^{2}$ Universidade Federal do Mato Grosso (UFMT) - Cuiabá, MT \\ ${ }^{3}$ Escola de Veterinária e Zootecnia - Universidade Federal de Goiás - Goiânia, GO \\ ${ }^{4}$ Universidade Federal Rural da Amazônia (UFRA), PA
}

\begin{abstract}
RESUMO
Descreveram-se os sinais clínicos e achados anatomopatológicos da intoxicação crônica por cobre em um ovino da raça Texxel e definiu-se a conduta diagnóstica correta para confirmação da enfermidade. Um ovino foi encaminhado ao setor de patologia com histórico de apatia, hemoglobinúria e morte em dois a três dias. No exame necroscópico, observaram-se icterícia e edema subcutâneo, fígado aumentado de volume e amarelado e rins escuros. No exame histológico, observaram-se necrose zonal aleatória e acentuada no fígado, necrose epitelial tubular, gotas hialinas e cilindros marrom-alaranjados em túbulos coletores dos rins. O histórico alimentar, a sensibilidade de espécie/raça, o quadro clínico, as alterações macroscópicas e microscópicas sugeriram o quadro de intoxicação crônica por cobre. A confirmação diagnóstica somente foi possível após a marcação de pigmentos de cobre pela técnica histoquímica de Ulzmann e pela quantificação de cobre em matéria seca de fígado e rins, cujos valores foram mais altos que o normal.
\end{abstract}

Palavras-chave: hemólise, hemoglobinúria, histoquímica, métodos analíticos, ovinos

\begin{abstract}
The present work describes the clinical signs and anatomopathological findings of chronic copper toxicities in a Texxel breed sheep and defines the optimal diagnostic procedure for confirmation of the disorder. A sheep was sent to pathology analysis service with a history of apathy, hemoglobinuria and death within two to three days. Necropsy showed jaundice and subcutaneous edema, enlarged yellow liver and dark kidneys. The histologic examination showed random zonal necrosis, marked necrosis in the liver and tubular epithelial and orange-brown spotted hyaline cylinders in the collecting tubules of the kidneys. The dietary history, sensitivity of species/breed, clinical, macroscopic and microscopic alterations suggested the framework of chronic copper poisoning. Diagnostic confirmation was only possible after staining copper pigments trough the Ulzmann technique and quantification of copper in the dry liver and kidney, which were higher than normal levels.
\end{abstract}

Keywords: analytical methods, hemolysis, hemoglobinuria, histochemical, sheep

\section{INTRODUÇÃO}

A ovinocultura no cerrado tem crescido expressivamente nas últimas décadas, principalmente devido à intensa produção de grãos em toda região Centro-Oeste. Porém, a maioria dos produtores desconhece as exigências e o manejo nutricional da espécie ovina. Um erro comum é empregar a mesma dieta mineral dos bovinos na criação de ovinos, desencadeando diversas desordens nutricionais e metabólicas, como a toxemia da prenhez, urolitíase e intoxicação por cobre (Ortolani, 2003; Silva, 2011).

Recebido em 21 de outubro de 2011

Aceito em 8 de outubro de 2012

Email: mapa_mi@hotmail.com 
O quadro de intoxicação por cobre pode apresentar-se de duas formas distintas: um quadro agudo, em que o animal apresenta gastrenterite logo após o consumo de alta quantidade de cobre (Lemos et al., 1997; Sargison, 2003), e uma forma crônica, em que ocorre o acúmulo gradativo do elemento em vários tecidos, principalmente no fígado, sem sinais clínicos durante alguns meses. Após uma situação de estresse, ocorre a liberação do elemento armazenado do fígado, causando quadro hemolítico e nefropatia (Riet-Correa et al., 1989; Lemos et al., 1997; Ortolani, 2003).

A intoxicação por cobre é uma das principais causas de mortalidade por intoxicação em ovinos (Rissi et al., 2010). Porém, os sinais clínicos e anatomopatológicos são similares aos de outras doenças, principalmente de intoxicação por braquiaria, sobretudo em animais criados em regime de pastoreio (Santos et al., 2008). Assim, vários casos tornam-se inconclusivos quando não são utilizadas técnicas analíticas no diagnóstico. Portanto, o objetivo deste trabalho é apresentar a conduta diagnóstica correta para confirmação de intoxicação crônica por cobre, além de descrever os sinais clínicos e achados anatomopatológicos em um ovino da raça Texxel.

\section{CASUÍSTICA}

Um ovino da raça Texxel, fêmea, quatro anos de idade, foi encaminhado ao setor de patologia. $\mathrm{O}$ animal é proveniente de um rebanho de 400 ovinos, criados semi-extensivamente em capim Brachiaria brizanta. Há aproximadamente oito meses, os ovinos eram suplementados com sal mineral destinado a bovinos. No rebanho existiam animais de ambos os sexos e com idade de três a cinco anos. $\mathrm{O}$ proprietário relatou que, nos últimos sete dias, cinco animais afastaram-se do grupo, tornaram-se apáticos, apresentaram urina vermelho-escura a amarronzada, entraram em decúbito esternal seguido de lateral e evoluíram para a morte. A evolução do quadro era de dois a três dias em média.
Na necropsia, observaram-se edema subcutâneo ventral discreto e pequena quantidade de líquido seroso nas cavidades abdominal e torácica. O fígado encontrava-se aumentado de volume, amarelado, com evidenciação do seu padrão lobular (Fig. 1A) e, à palpação, acentuadamente friável. Os rins exibiam coloração marrom-escura (Fig. 1B) e marcante aumento da estriação cortical e medular (Fig. 1D). O conteúdo da bexiga era marrom (450 mL) (Fig. 1C). Coletaram-se amostras de fígado, rins, coração, pulmões, músculos esqueléticos e encéfalo para fixação em formol a $10 \%$; amostras de fígado (400g), rins (250g) e conteúdo da bexiga $(450 \mathrm{~mL})$ foram refrigerados.

Os tecidos fixados em formol foram desidratados e diafanizados até a confecção dos blocos de parafina. Cortes de $5 \mu \mathrm{m}$ foram corados pelas técnicas histoquímicas de hematoxilina e eosina (HE) e de Ulzmann (Luna, 1968). No exame histológico, o fígado exibia áreas de necrose sem padrão zonal, irregulares, multifocais e acentuadas (Fig. 2A). O rim apresentava necrose epitelial tubular aguda, gotas hialinas e cilindros marrom-alaranjados (cilindros de hemoglobina) nos túbulos coletores (Fig. 2B). A coloração de Ulzmann confirmou a presença de cobre no tecido hepático, caracterizado por acúmulo de material amorfo de coloração castanhoenegrecida no citoplasma de hepatócitos (Fig. 2C) e de células de Kupffer.

As amostras refrigeradas foram encaminhadas ao Centro de Pesquisa de Alimentos para realização de análises físico-química e dosagem de cobre. As amostras foram trituradas, pesadas, secas em estufa e, após a secagem, pesadas novamente para cálculo da matéria seca. A concentração de cobre foi determinada por espectofotometria de absorção atômica e os resultados foram expressos com base na quantidade de matéria seca do tecido (Ortolani, 2003; Minervino et al., 2009). A análise físico-química quantificou $1850 \mathrm{mg} / \mathrm{kg}$ de peso seco (ps) de cobre na amostra de fígado, $72 \mathrm{mg} / \mathrm{kg}$ ps na amostra de rim e $6 \mathrm{mg} / \mathrm{kg}$ ps na amostra de urina. 


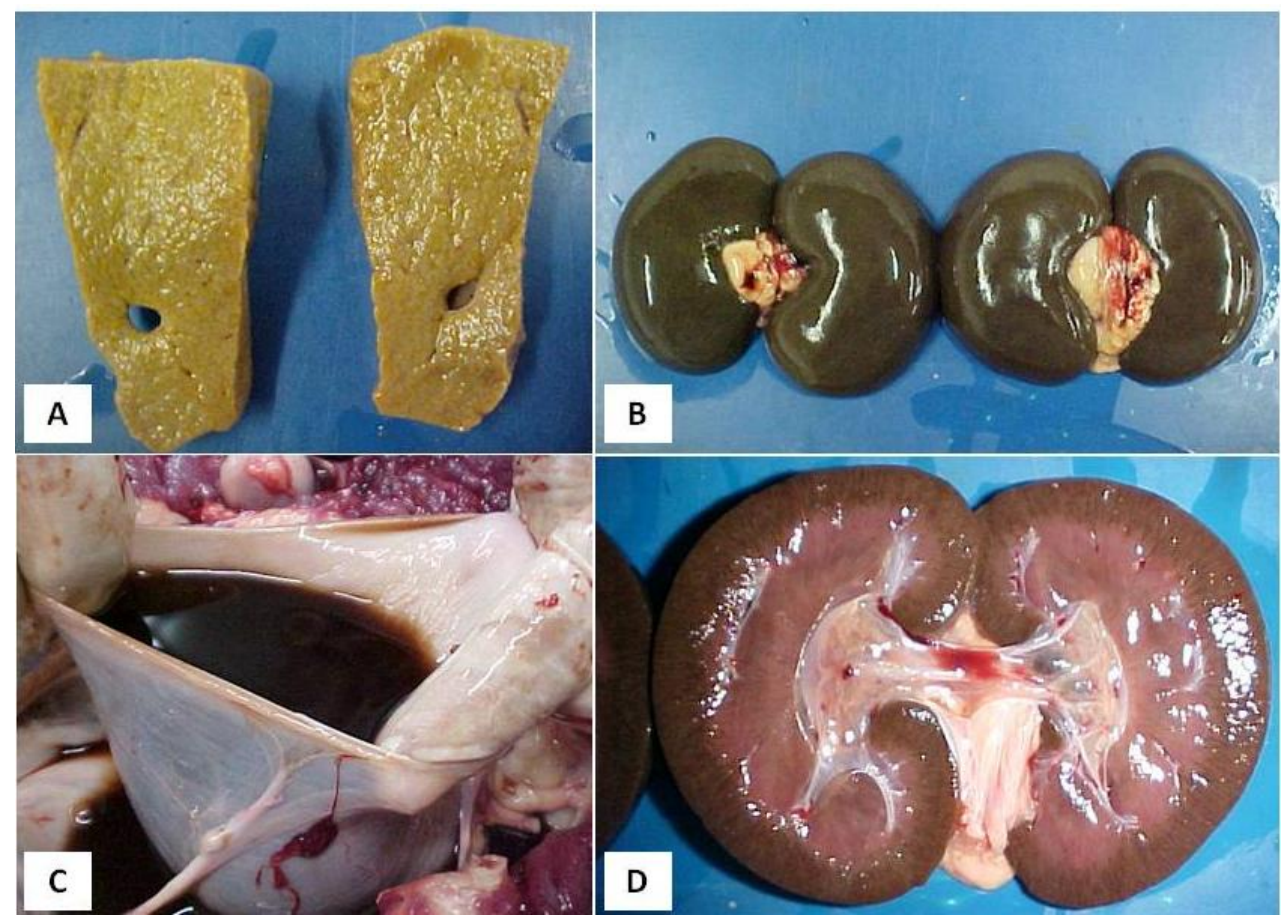

Figura 1. Ovino. A: fígado: acentuadamente amarelo com evidenciação do padrão lobular. B: rins: coloração marrom. C: bexiga: conteúdo marrom escuro. D: rim: aumento da estriação cortical.

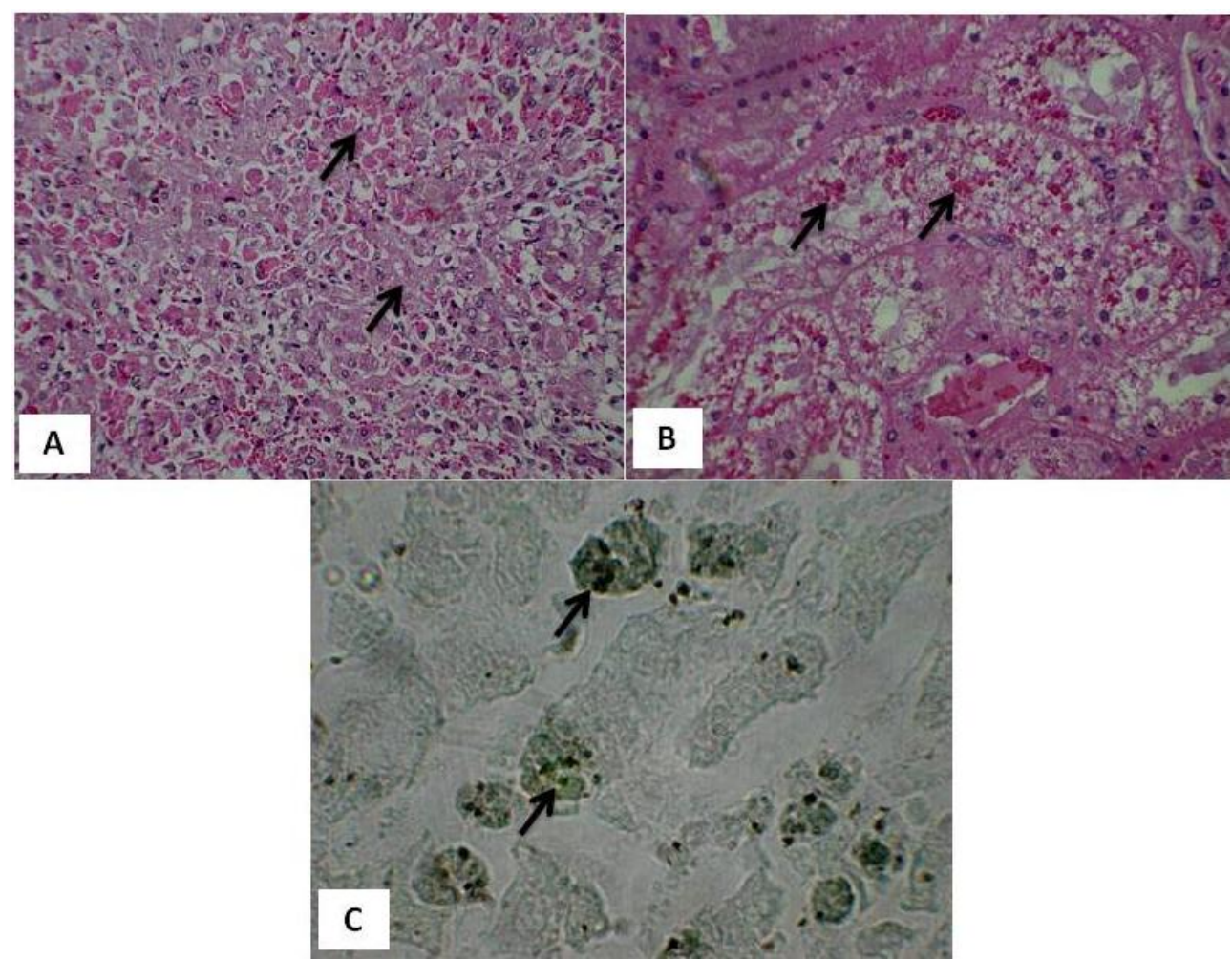

Figura 2. Fotomicrografias, ovino. A: fígado: áreas de necrose sem padrão zonal (setas), irregulares, multifocais e acentuadas, HE, obj. 10x. B: rim: necrose epitelial tubular aguda, gotas hialinas (setas) nos túbulos coletores, HE, obj. 20x. C: fígado: hepatócitos com depósito de cobre (setas), Ulzmann, obj. 40x. 


\section{DISCUSSÃO}

Os sinais clínicos do ovino deste relato eram, principalmente, apatia e hemoglobinúria. Esses mesmos sinais foram descritos por Riet-Correa $e t$ al. (1989), Méndez (2001), Gaetke e Chow (2003), Ortolani (2003), Oruc et al. (2009), Rissi et al. (2010). Há aproximadamente oito meses, os ovinos da propriedade receberam sal mineral para bovinos. Rosa e Gomes (1982); Riet-Correa et al. (1989), Méndez (2001), Ortolani (2003) consideram que o uso de sal mineral para bovinos na dieta de ovinos é uma das principais causas da intoxicação crônica por cobre.

Neste caso, a mistura mineral bovina continha $1.250 \mathrm{mg} / \mathrm{kg}$ de cobre. Rosa e Gomes (1982) afirmam que ovinos consomem, em média, $11 \mathrm{~g}$ dessa mistura por dia, o que corresponderia, neste caso, a $13,75 \mathrm{mg} / \mathrm{kg}$ de cobre. De acordo com Ortolani (2003), o requisito de cobre diário para ovinos varia de 5 a $12 \mathrm{mg} / \mathrm{kg}$. Já Ribeiro et al. (2007) afirmam que essa espécie não tolera dietas que contenham mais de $10 \mathrm{mg} / \mathrm{kg}$ de cobre. Radostis et al. (2002) alertam que $3,5 \mathrm{mg} / \mathrm{kg}$ de cobre na dieta acima da exigência diária induz aos quadros de intoxicação crônica em ovinos. No presente caso, é possível que a quantidade de cobre na dieta fosse superior a $13,75 \mathrm{mg} / \mathrm{kg}$ devido à provável presença desse mineral no solo e na gramínea de $B$. brizanta.

Soares (2004) explica que ovinos absorvem o cobre da dieta de acordo com a quantidade oferecida, não havendo restrição de sua quantidade, diferentemente do que ocorre na absorção de outros minerais. Após sua absorção, o cobre é direcionado ao fígado, onde ocorre o acúmulo do elemento, podendo alcançar níveis tóxicos após meses ou anos. Desta forma, o histórico de alimentação contendo sal mineral bovino descrito pelo proprietário foi fundamental para a suspeita diagnóstica. Além disso, ovinos da raça Texxel, de acordo com Ortolani (2003), apresentam maior sensibilidade à intoxicação por cobre.

$\mathrm{Na}$ intoxicação crônica por cobre, os achados macroscópicos dependem da evolução do quadro clínico-patológico, mas, nos casos relatados por Riet-Correa et al. (1989), Méndez (2001), Ortolani (2003), Soares (2004), Castro et al. (2007), Ribeiro et al. (2007), Headley et al. (2008), Giadinis et al. (2009), Oruc et al. (2009),
Rissi et al. (2010), as alterações encontradas foram semelhantes às descritas neste caso. As lesões microscópicas observadas no ovino deste caso ocorreram principalmente no fígado e nos rins, assim como relatado por Riet-Correa et al. (1989), Mendéz (2001), Soares (2004), Rissi et al. (2010), em relatos de intoxicação crônica por cobre. No entanto, os achados macroscópicos e microscópicos não foram suficientes para a conclusão do caso, haja vista que são alterações encontradas em outras enfermidades, como, por exemplo, intoxicação por Brachiaria spp e intoxicação por chumbo.

Após avaliação do histórico e leitura das lâminas em HE, solicitou-se uma coloração específica para marcação de cobre, neste caso, a coloração de Ulzmann, que foi positiva para identificação do mineral. Riet-Correa et al. (1989), Mendéz (2001) e Soares (2004) sugerem esta coloração para confirmação de acúmulo de cobre hepático. Desta forma, a confirmação anatomopatológica do caso somente foi possível pela marcação do mineral cobre no tecido hepático.

Por se tratar de suspeita de intoxicação, é conduta básica a coleta de material para detecção e quantificação do elemento mineral. Neste caso, os níveis de cobre foram superiores a $500 \mathrm{mg} / \mathrm{kg}$ ps no fígado $(1850 \mathrm{mg} / \mathrm{kg} \mathrm{ps})$, como descrito por Riet-Correa et al. (1989), Soares (2004), Mendéz (2001), em quadros de intoxicação por cobre, e superiores a $26-61 \mathrm{mg} / \mathrm{kg}$ ps no rim $(72 \mathrm{mg} / \mathrm{kg} \mathrm{ps})$, como encontrado por Oruc et al. (2009) e Gooneratne et al. (1980). É importante ressaltar que a análise toxicológica é ferramenta diagnóstica fundamental para a confirmação da intoxicação, pois permite a identificação e, principalmente, a quantificação do elemento suspeito (Lemos et al., 1997).

\section{CONCLUSÕES}

O emprego da coloração de Ulzmann e a quantificação de cobre por métodos analíticos em tecidos de animais sob pastejo foram cruciais para a confirmação do quadro de intoxicação crônica por cobre, sobretudo, pela diferenciação da intoxicação por Brachiaria spp. Portanto, análises histoquímicas específicas para marcação de cobre e a quantificação toxicológica devem fazer parte da conduta diagnóstica nos casos suspeitos de intoxicação por cobre. Além disso, o histórico alimentar, a sensibilidade de 
espécie/raça, o quadro clínico, as alterações macroscópicas e microscópicas e os dados epidemiológicos auxiliaram na suspeita desse diagnóstico. A insuficiência hepática e a renal aguda e a anemia hemolítica causaram a morte do ovino.

\section{REFERÊNCIAS}

CASTRO, M.B.; CHARDULO, L.A.L.; SZABÓ, M.P.J. Copper toxicosis in sheep fed dairy cattle ration in São Paulo, Brazil. Arq. Bras. Med. Vet. Zootec., v.59, p.246-249, 2007.

GAETKE, L.M.; CHOW, C.K. Copper toxicity, oxidative stress, and antioxidant nutrients. Toxicology, v.189, p.147-163, 2003.

GIADINIS, N.D.; PAPAIOANNOU, N.; KRITSEPIKONSTANTINOU, M. et al. Acute encephalopathy and clinical pathology findings in a sheep with chronic copper poisoning, Turk. J. Vet. Anim. Sci., v.33, p.363366, 2009.

GOONERATNE, S.R.; HOWELL, McC.J.; COOK, R.D. An ultrastructural and morphometric study of the liver of normal and copper-poisoned sheep. Am. $J$. Pathol., v.99, p.429-450, 1980.

HEADLEY, S.A.; REIS, A.C.F.; DUIM, T.L. et al. Chronic copper poisoning in sheep from nothern Paraná, Brazil: case report. Semina: Cienc. Agrar., v.29, p.179-184, 2008.

LEMOS, R.A.A.; RANGEL, J.M.R.; OSÓRIO, A.L.A.R. et al. Alterações clínicas, patológicas e laboratoriais na intoxicação crônica por cobre em ovinos. Cienc. Anim., v.27, p.457-463, 1997.

LUNA, L.G. (Ed). Manual of histological staining methods of the Armed Forces Institute of Pathology. Washington: Mc Graw Hill, 1968. 258p.

MÉNDEZ, M.C. Intoxicação crônica por cobre. In: RIET-CORREA, F.; SCHILD, A.L.; MÉNDEZ, M.C. et al. (Ed). Doenças de ruminantes e equinos. 2.ed. São Paulo: Livraria Varela, 2001. p.181-186.

MINERVINO, A.H.H.; BARRÊTO JR, R.A.; FERREIRA, R.N.F. et al. Intoxicação cúprica acumulativa em búfalos. Semina: Cienc. Agrar, v.30, p.407-416, 2009.

ORTOLANI, E.L. Intoxicação cúprica acumulativa em ovinos. In: CONGRESSO LATINO AMERICANO, 11., 2003, Salvador. Anais... Salvador: Sociedade Brasileira de Buiatria, 2003. p.113-114. (Resumo).
ORUC, H.H.; CENGIZ, M.; BESKAYA, A. Chronic copper toxicosis in sheep following the use of copper sulfate as a fungicide on fruit trees. J. Vet. Diagn. Invest., v.21, p.540-543, 2009.

RADOSTIS, O.M.; GAY, C.C.; BLOOD, D.C. et al. Doenças causadas por substâncias químicas inorgânicas e produtos químicos. In:__.Clínica Veterinária - Um Tratado de Doenças dos Bovinos, Ovinos, Suínos, Caprinos e Equinos. 9.ed. Rio de Janeiro: Guanabara Koogan, 2002. p.1417-1471.

RIBEIRO, L.A.O.; RODRIGUES, N.C.; SMIDERLE, W.A. Intoxicação crônica por cobre em ovelhas pastoreadas em pomar de videiras adubado com cama aviária. Braz. J. Vet. Res. Anim. Sci., v.44, p.208-211, 2007.

RIET-CORREA, F.; OLIVEIRA, J.A.; GIESTA, S. et al. Intoxicação crônica por cobre em ovinos no Rio Grande do Sul. Pesq. Vet. Bras., v.9, p.51-54, 1989.

RISSI, D.R.; PIEREZAN, F.; OLIVEIRA FILHO, J.C. et al. Doenças de ovinos da região Central do Rio Grande do Sul: 361 casos. Pesq. Vet. Bras., v.30, p.21$28,2010 . j$

ROSA, I.V.; GOMES, R.F. Intoxicação crônica por cobre. Comun. Téc. 14, Embrapa-CNPGC, p.1-3, 1982.

SANTOS, J.C.A.; RIET-CORREA, F.; SIMÕES, S.V.D. et al. Patogênese, sinais clínicos e patologia das doenças causadas por plantas hepatotóxicas em ruminantes e equinos no Brasil. Pesq. Vet. Bras., v.28, p.1-14, 2008.

SARGISON, N. Copper poisoning. NADIS, 2003. Disponível em: <http://www.mlc. org.uk/sheep/technical/>. Acessado em: 15 out. 2004.

SILVA, R.A.M.S. Integração bovinos-ovinos e a produção de cordeiros como alternativa econômica para o Pantanal. Informativo da Cadeia da Carne Bovina do Pantanal Mato-Grossense, 2011. Disponível em: <http://www.cpap.embrapa.br/cadeia carne/Online/CC011.pdf>. Acessado em: 03 set. 2011.

SOARES, P.C. Efeitos da intoxicação cúprica e do tratamento com tetratiomolibdato sobre a função renal e o metabolismo oxidativo de ovinos. 2004. 117f. Tese (Doutorado em Medicina Veterinária) Faculdade de Medicina Veterinária e Zootecnia, Universidade de São Paulo, São Paulo. 